\title{
Cannabinoid hyperemesis syndrome: an underreported entity causing nausea and vomiting of pregnancy
}

\author{
Seraina M. Schmid • Olav Lapaire • \\ Dorothy J. Huang · Frank Edwin Jürgens • \\ Uwe Güth
}

Received: 1 October 2010/Accepted: 6 December 2010/Published online: 18 December 2010

(C) Springer-Verlag 2010

\begin{abstract}
Introduction In the western world, cannabis is the most widely used drug of abuse. Cannabinoid hyperemesis syndrome, which seems to be a rare paradoxical reaction in individuals with a particular predisposition, is characterized by cyclic severe nausea and vomiting in long-term cannabis users. While the symptoms are unresponsive to antiemetic drugs, compulsive hot baths result in a considerable symptom relief.

Methods We report the first case of cannabinoid hyperemesis syndrome in pregnancy. A 26-year-old patient was admitted to our clinic in the 10th week of gestation.

Conclusion Before undertaking time-consuming and expensive medical examinations to rule out other medical reasons for therapy-resistant hyperemesis in pregnancy, obstetricians should determine whether compulsive bathing or showering provides symptomatic relief and ask specific questions regarding possible/suspected cannabis consumption.
\end{abstract}

Keywords Cannabis - Hyperemesis - Pregnancy ·

Vomiting

\section{Introduction}

In the Western world, cannabis is the most widely used illicit dependence causing substance and after the legal

S. M. Schmid · O. Lapaire · D. J. Huang · U. Güth ( ()

Department of Gynecology and Obstetrics,

University Hospital Basel (UHB), Spitalstrasse 21,

4031 Basel, Switzerland

e-mail: ugueth@uhbs.ch

F. E. Jürgens

Department for Addiction Medicine, LWL Clinic Gütersloh, Hermann-Simon-Straße 7, 33334 Gütersloh, Germany drugs tobacco and alcohol the third most commonly used one. In Europe, the prevalence of cannabis use among young adults has increased from an average of 5\% in 1990 to $15 \%$ in 2005 [1]. According to estimates, approximately $3-10 \%$ of pregnant women regularly consume cannabis [2].

In 2004, Allen et al. [3] first described a cannabinoid hyperemesis syndrome (CHS). The clinical symptoms include cyclical vomiting patterns. We report the first case to our knowledge of this entity in pregnancy. We discuss this syndrome in the particular context of pregnancy and hope to increase the awareness of the obstetricians of this unusual and underreported situation.

\section{Case report}

A 26-year-old Caucasian woman presented to the University Women's Hospital Basel (Basel, Switzerland) in the 10th week of gestation with nausea and vomiting for the past $24 \mathrm{~h}$. On admission, the patient reported a prior hospitalization 4 months ago in the general emergency department due to gastritis-like symptoms with nausea and vomiting. The further medical history revealed an earlier termination of pregnancy 7 years ago and knee surgery 3 years ago. Except for smoking five cigarettes a day, she reported no drug use or medication intake.

Transvaginal sonography showed a singleton pregnancy consistent with 10 gestational weeks. The ovaries were unremarkable. Hyperemesis gravidarum was assumed and the standard laboratory workup of urine and blood samples was performed. The only abnormal parameter was significant ketonuria. A normal thyroid-stimulating hormone (TSH) level excluded primary or pregnancy-induced hyperthyroidism. 
During the first days of the inpatient stay, the patient suffered from frequent and severe vomiting episodes (up to 12 times a day) and nearly constant nausea. The symptoms were resistant to the administration of vitamin B6, metoclopramide, chlorazine, dexamethasone and ondansetron. Dehydration was treated by intravenous fluid replacement. On the second day of hospitalization, the medical staff noted that the patient showered more than once a day and her constitution and mood seemed to considerably improve during and shortly after showering. Because of this additional information, we began to consider CHS. After specific inquiry, the patient admitted to regular daily use of cannabis since the age of 13. She had only stopped smoking cannabis after learning about her pregnancy, which was about 10 days before the onset of the current symptoms. The subsequently conducted urine drug screen was positive for cannabis, but not for any other drug. With the presumptive diagnosis of CHS, we suggested taking hot baths and showers. With these measures, her symptoms improved considerably and we stopped all antiemetic medication. The patient was discharged a day later and was afterwards regularly seen in our outpatient clinic. She continued taking hot baths and showers up to five times a day, which led to a massive improvement of her symptoms and the recovery of her appetite. Two weeks later, all symptoms were resolved.

Looking back, the patient reported that she had experienced recurrent episodes (about twice a year) of nausea and vomiting over the last 3.5 years without having altered her cannabis consumption. She was even once hospitalized in the psychiatric clinic due to vomiting which was considered psychogenic. During one of these first episodes she experienced improvement of her symptoms during and after taking hot showers or baths. Having stopped cannabis use immediately after learning about her early pregnancy and knowing of the high frequency of morning sickness in pregnancy, it did not occur to the patient that there could be a connection between the actual symptoms and the ones she repetitively experienced over the previous years. Over time the patient stayed abstinent and remained symptom free. The pregnancy progressed without further complications.

\section{Comment}

Since there are no larger scale systematic analyses of CHS to date, the typical clinical features for this syndrome were established based on isolated case reports published in the last 7 years [3-14] (Table 1). After regular cannabis use for several years, the patients develop severe nausea and vomiting, often accompanied by colicky abdominal pain without an obvious organic cause; this recurs in a cyclic pattern over months. While the symptoms are unresponsive
Table 1 Diagnostic features of cannabinoid hyperemesis syndrome (modified from [12])

Essential precondition

History of regular cannabis use for years

Clinical features

Severe nausea and vomiting, unresponsive to antiemetic agents

Vomiting, often accompanied by colicky abdominal pain that recurs in a cyclic pattern of months

Compulsive hot baths/showers with considerable symptom relief

No evidence of other underlying gastrointestinal (e.g. gall bladder or pancreatic inflammation), genitourinary, metabolic or neurological diseases which could cause similar clinical symptoms

Resolution of symptoms after stopping cannabis use

to the common antiemetic drugs, taking hot baths or showers improves the condition. Acquired compulsive bathing behavior, a result of this unconventional "therapy", is thus a notable characteristic of CHS. Normally, the symptoms resolve in $24-48 \mathrm{~h}$ with conservative management and cessation of cannabis use. As described in our case, the symptoms may also occur after several days to a few weeks of cannabis abstinence.

Since cannabis is known to have an antiemetic effect which can be particularly useful in the treatment of cancer patients [15], CHS seems to be a rare paradoxical reaction in individuals with a particular predisposition. The mechanism of CHS is unknown. Theories attempting to explain both the symptoms and the effectiveness of the unusual bathing "therapy", include dose-dependent build-up of cannabinoids and effects of agent-related toxicity, as well as alterations of cannabinoid receptors in the brain, in particular, in the hypothalamus which regulates thermoregulation and autonomic equilibrium via the limbic system $[3,7,12]$.

Previously published case reports regarding CHS come exclusively from internal medicine/gastroenterology [3-12, 14] or psychiatry/addiction medicine [13]. We present the first case of this entity in connection with pregnancy. Compared to the above-mentioned medical subspecialities, there are relevant differences in obstetrics:

- In gastroenterology and particularly in psychiatry/ addiction medicine, the intake of illicit drugs is often frankly reported by the patients, which is not always the case in obstetrics. The hazards of drug abuse in pregnancy are widely known [16]. Although moderate drug use is not only accepted, but even in vogue in some social circles, drug abuse during pregnancy is objectionable. This may lead to pregnant patients intentionally concealing their drug consumption to avoid uncomfortable situations. One should be aware that overtly critical or hostile behavior of the medical 
staff towards pregnant drug users may act as a barrier to compliance with surveillance and treatment. Furthermore, some patients may not associate their complaints with illicit drug use, as in the case presented here, and feel that consumption does not need to be mentioned at all.

- Particularly in early pregnancy, hyperemesis gravidarum is a much more common and plausible diagnosis for excessive nausea and vomiting. However, resistance to antiemetic therapy is suspicious for CHS: while hyperemesis gravidarum usually responds to medical therapy (nonpharmacologic and antiemetic drugs such as $\mathrm{H}_{1}$ blockers, dopamine antagonists, phenothiazines, $5-\mathrm{HT}_{3}$ receptor agonists and steroids) [17], administration of these agents does not lead to symptomatic relief in patients with CHS.

Therefore, before undertaking time-consuming and expensive medical examinations to rule out other medical reasons for therapy-resistant hyperemesis (e.g., gastrointestinal and genitourinary conditions, metabolic and neurologic disorders) [17], obstetricians should perform the following:

1. Determine whether compulsive bathing provides symptomatic relief (pathognomonic feature of CHS).

2. Ask specific questions regarding possible/suspected cannabis consumption.

Should the patient admit to drug abuse, an interdisciplinary approach to the problem, including the involvement of experienced drug counselors, should be immediately undertaken.

Conflict of interest None.

\section{References}

1. European Monitoring Centre of Drugs and Drug Addiction (2006) Annual report 2006: the state of the drugs problem in
Europe. Report No.: 92-9168-266-7. Office for Official Publications of the European Communities, Luxembourg

2. Lamy S, Thibaut F (2010) Psychoactive substance use during pregnancy: a review. Encephale 36(1):33-38

3. Allen JH, de Moore GM, Heddle R, Twartz JC (2004) Cannabinoid hyperemesis: cyclical hyperemesis in association with chronic cannabis abuse. Gut 53(11):1566-1570

4. Alfonso Moreno V, Ojesa F, Moreno-Osset E (2006) Cannabinoid hyperemesis. Gastroenterol Hepatol 29(7):434-435

5. Boeckxstaens GE (2005) Cannabinoid hyperemesis with the unusual symptom of compulsive bathing. Ned Tijdschr Geneeskd 149(26):1468-1471

6. Budhraja V, Narang T, Azeez S (2008) Cannabinoid hyperemesis syndrome: cyclic vomiting, chronic cannabis use, and compulsive bathing. Pract Gastroenterol 32(9):79-80

7. Chang YH, Windish DM (2009) Cannabinoid hyperemesis relieved by compulsive bathing. Mayo Clin Proc 84(1):76-78

8. Chepyala P, Olden KW (2008) Cyclic vomiting and compulsive bathing with chronic cannabis abuse. Clin Gastroenterol Hepatol 6(6):710-712

9. Ochoa-Mangado E, Jimenez Gimenez M, Salvador Vadillo E, Madoz-Gurpide A (2009) Cyclical hyperemesis secondary to cannabis abuse. Gastroenterol Hepatol 32(6):406-409

10. Roelofs J, Vorel SK, Vorel-Havelkova E, Brombacher PJ (2005) Cannabinoid hyperemesis with the unusual symptom of compulsive bathing. Ned Tijdschr Geneeskd 149(42):2376

11. Singh E, Coyle W (2008) Cannabinoid hyperemesis. Am J Gastroenterol 103(4):1048-1049

12. Sontineni SP, Chaudhary S, Sontineni V, Lanspa SJ (2009) Cannabinoid hyperemesis syndrome: clinical diagnosis of an underrecognised manifestation of chronic cannabis abuse. World J Gastroenterol 15(10):1264-1266

13. Wallace D, Martin AL, Park B (2007) Cannabinoid hyperemesis: marijuana puts patients in hot water. Australas Psychiatry $15(2): 156-158$

14. Watts M (2009) Cannabinoid hyperemesis presenting to a New Zealand hospital. N Z Med J 122(1290):116-118

15. Walsh D, Nelson KA, Mahmoud FA (2003) Established and potential therapeutic applications of cannabinoids in oncology. Support Care Cancer 11(3):137-143

16. Keegan J, Parva M, Finnegan M, Gerson A, Belden M (2010) Addiction in pregnancy. J Addict Dis 29(2):175-191

17. ACOG (American College of Obstetrics, Gynecology) (2004) Practice bulletin: nausea and vomiting of pregnancy. Obstet Gynecol 103(4):803-814 